\title{
Phase locked fluxon-antifluxon states in stacked Josephson junctions
}

Carapella, Giovanni; Constabile, Giovanni; Petraglia, Antonio; Pedersen, Niels Falsig; Mygind, Jesper

Published in:

Applied Physics Letters

Link to article, DOI:

$10.1063 / 1.117398$

Publication date:

1996

Document Version

Publisher's PDF, also known as Version of record

Link back to DTU Orbit

Citation (APA):

Carapella, G., Constabile, G., Petraglia, A., Pedersen, N. F., \& Mygind, J. (1996). Phase locked fluxon-antifluxon states in stacked Josephson junctions. Applied Physics Letters, 69(9), 1300-1302.

https://doi.org/10.1063/1.117398

\section{General rights}

Copyright and moral rights for the publications made accessible in the public portal are retained by the authors and/or other copyright owners and it is a condition of accessing publications that users recognise and abide by the legal requirements associated with these rights.

- Users may download and print one copy of any publication from the public portal for the purpose of private study or research.

- You may not further distribute the material or use it for any profit-making activity or commercial gain

- You may freely distribute the URL identifying the publication in the public portal 


\title{
Phase locked fluxon-antifluxon states in stacked Josephson junctions
}

\author{
G. Carapella, ${ }^{\text {a) }}$ G. Costabile,$^{\text {a) }}$ A. Petraglia, ${ }^{\text {b) }}$ N. F. Pedersen, and J. Mygind \\ Department of Physics, Building 309, Technical University of Denmark, DK-2800 Lyngby, Denmark
}

(Received 25 April 1996; accepted for publication 17 June 1996)

\begin{abstract}
Measurements were made on a two-stack long Josephson junction with very similar parameters and electrical access to the thin middle electrode. Mutually phase-locked fluxon-antifluxon states were observed. The observed propagation velocity is in agreement with the theoretical prediction. The $I-V$ curves recorded using the bias current in one of the junctions as fixed parameter show that these states continue to also exist when the other junction is oppositely biased. This is found in numerical simulations based on the experimental junction parameters as well. We propose a model in which waves in the junctions coexist with fluxons. (C) 1996 American Institute of Physics. [S0003-6951(96)02635-6]
\end{abstract}

In recent years a great deal of interest has been devoted to stacked long Josephson junctions. Experiments ${ }^{1-3}$ have demonstrated that it is possible to phase lock the fluxon motion in stacked junctions. This may lead to a new family of Josephson junction high frequency microwave oscillators. Numerical simulations ${ }^{4,5}$ as well as analytical calculations ${ }^{6}$ have shown rich dynamics. It is difficult to fabricate stacked junctions that have the same parameters, and where one can connect current and voltage leads to the intermediate superconducting layers ${ }^{7}$.

Here we report on new measurements on two-stack long Josephson junctions with very similar junction properties, and with electrical connections to the middle electrode. Figure 1 shows the overlap geometry and the convention used for the directions of the bias currents. The $\mathrm{Nb}-\mathrm{Al}_{2} \mathrm{O}_{3}-\mathrm{Nb}-\mathrm{Al}_{2} \mathrm{O}_{3}-\mathrm{Nb}$ samples were fabricated at the University of Salerno, Italy, and measured at the Technical University of Denmark, Denmark. The experiments with this strongly nonlinear system have demonstrated a very rich dynamics, and we have found states not observed earlier. For clarity we only present results obtained on a single sample with dimensions $L \times W=600 \mu \mathrm{m} \times 20 \mu \mathrm{m}$. The top and bottom electrodes are $3000 \AA$ thick while the middle electrode is only about $600 \AA$ thick, giving rise to a strong coupling between the two junctions. The relatively large subgap current and the corresponding large damping given by the fabrication process were advantageous to the experiments.

Typical experimental results are shown in Fig. 2. The $I-V$ curves show a series of a zero field steps (ZFS) in the bottom junction (junction $B$ ) recorded with the current in the top junction (junction A) as a parameter in the situation where the two junctions are voltage locked. We note that for a two-stack junction there are two different propagation velocities for small-amplitude electromagnetic waves ${ }^{1,2,4,6}, c^{+}$ and $c^{-}$. Those velocities were determined as was done in Ref. 1 by measuring the voltage spacing of the Fiske steps obtained with a magnetic field applied perpendicular to $L$ in the plane of the junction. The voltage spacing of the $c^{-}$ Fiske step series was $9.7 \mu \mathrm{V}$ while that of the $c^{+}$series was $18.4 \mu \mathrm{V}$. This identifies the ZFS series in Fig. 2 as zero field

\footnotetext{
${ }^{a)}$ Permanent address: Universitá di Salerno, Dipartimento di Fisica, I-84081 Baronissi (SA), Italy.

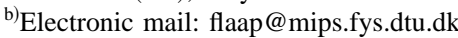

steps belonging to the $c^{-}$mode with an asymptotic voltage twice that of the corresponding Fiske step series, i.e., $\sim 19.5$ $\mu \mathrm{V}$. Since the biasing of the two junctions corresponds to a fluxon in one junction and an antifluxon in the other, the ZFS in Fig. 2 reflects a phase-locked fluxon-antifluxon zero field step. To our knowledge this type of excitation has not been observed before. A particular feature of Fig. 2 is that the current of the ZFS may become negative while the voltage is positive. This apparent contradiction is in agreement with theory as will be demonstrated below. In the inset of Fig. 2 we plot all the curves using a current axis defined by $\left(I_{A}+I_{B}\right) / 2$, and the curve where the currents in the two junctions are the same; it is clear that all the curves fall on top of each other. The origin of the small resonance and of the instability region in the lower part of some of the ZFSs is not yet clear. However, noticing that it is enhanced in the curves relative to the largest value of $I_{A}-I_{B}$, we suggest that it may be associated to the relative oscillations of the fluxonantifluxon pair.

We note that in addition to the types of ZFS shown in Fig. 2 we also observe the $n=2,3,4$, and 5 curves. For these curves there was locking between 2, 3, 4, and 5 fluxons in one junction and 2, 3, 4, and 5 antifluxons in the other junction. We also observed $n=-1$ and -2 step, i.e., with negative voltage. Careful examination of the voltages in the two junctions revealed that this corresponds to an antifluxonfluxon excitation, as was confirmed by a numerical simulation with the approximate experimental parameters. Figure 3

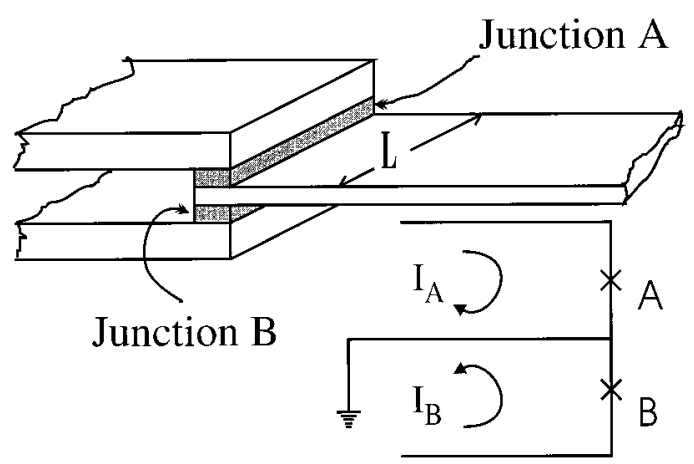

FIG. 1. Schematic drawing of the stack used; the positive direction for the bias currents is indicated by arrows. 


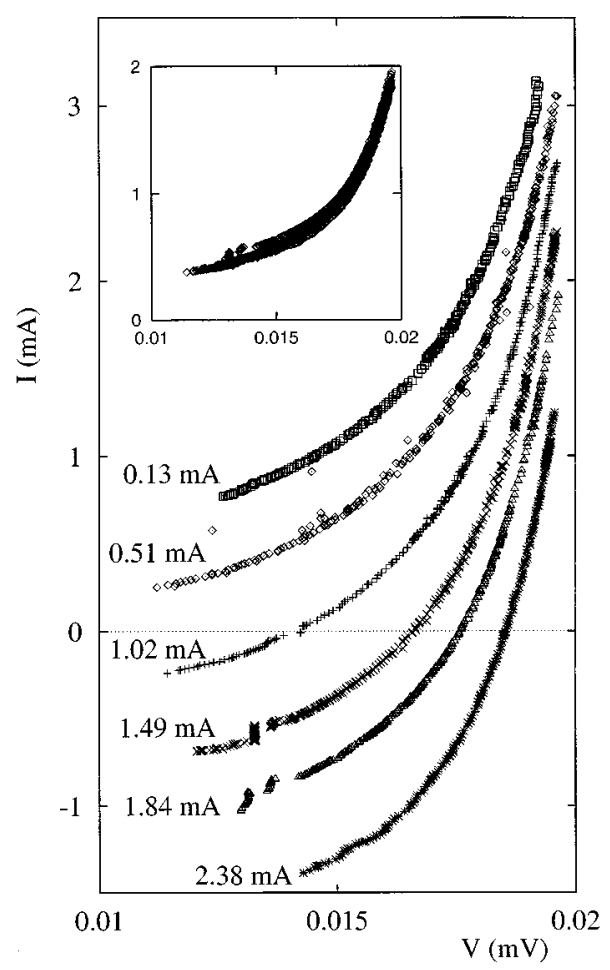

FIG. 2. Current-voltage curves at $T=4.2 \mathrm{~K}$ for the first zero field step of the bottom junction in the voltage locked state. Each profile is obtained with a different value of the bias current in the other junction. In the inset the same curves are represented putting the average bias current $\left(I_{A}+I_{B}\right) / 2$ on the $y$ axis.

shows the range of stability of the fluxon-antifluxon phase locking in the $I_{A}, I_{B}$ plane. As can be seen, it extends over three quadrants, corresponding to the steps shown in Fig. 2. If the antifluxon-fluxon steps corresponding to negative voltage are included we have a similar region symmetric with the shown area along the line $I_{A}=-I_{B}$. The inset shows the numerical result for the same mode; the model used is described below, and the parameters are given in the caption; the similarity is striking. We have based the evaluation of the

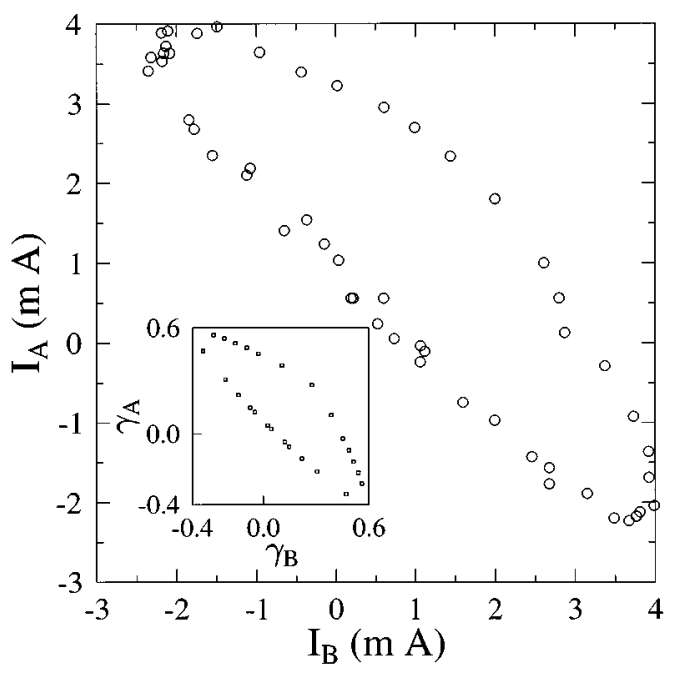

FIG. 3. Range of stability for the fluxon-antifluxon mode in the currents parameter space. Inset: numerical results with parameters $\alpha=0.1, l=10$, $s=0.5$. system on the experimental parameters. The initial slope of the $I$ - $V$ curve of the ZFS gives a normalized damping parameter of $\alpha \sim 0.05$. The maximum measured switching current was $4.5 \mathrm{~mA}$, the normalized length (defined as $\left.l=L / \lambda_{J}\right)$ was $l \sim 10$. Since $c^{ \pm}=\overline{c l} \sqrt{1 \pm s}$ we determine a coupling parameter of $s=-0.56$. This is consistent with the theoretical expression given in Ref. 4 and the known thickness of the middle film and the London penetration depth.

The coupled sine-Gordon equations for the geometry in Fig. 1 are $^{6}$

$$
\left(\begin{array}{l}
\varphi_{x x} \\
\psi_{x x}
\end{array}\right)=\left(\begin{array}{ll}
1 & s \\
s & 1
\end{array}\right)\left(\begin{array}{l}
J_{A} \\
J_{B}
\end{array}\right)-(1+s)\left(\begin{array}{c}
\gamma_{A} \\
-\gamma_{B}
\end{array}\right) ;
$$

here $\varphi$ and $\psi$ are the phase differences for the two junctions and $J_{A}$ and $J_{B}$ the currents through layers $A$ and $B$. The negative sign in front of $\gamma_{B}$ is a consequence of the sign convention introduced in connection with Fig. 1; we assume the two solutions to be (see Ref. 8)

$$
\begin{aligned}
& \varphi=\varphi^{I}+\varphi^{S}, \\
& \psi=\varphi^{I}-\varphi^{S} .
\end{aligned}
$$

Here $\varphi^{I}$ is the in-phase part, and $\varphi^{S}$ is the out-of-phase (symmetric) part of the solution (in that convention a fluxonfluxon phase-locked solution is described only by the term $\varphi^{I}$ while a fluxon-antifluxon phase-locked solution is described by $\varphi^{S}$ ). Inserted in Eq. (1) this gives the following equations

$$
\begin{aligned}
& -\frac{\varphi_{x x}^{I}}{1+s}+\varphi_{t t}^{I}+\alpha \varphi_{t}^{I}+\sin \varphi^{I} \cos \varphi^{S}=\frac{\gamma_{A}-\gamma_{B}}{2} \\
& -\frac{\varphi_{x x}^{S}}{1-s}+\varphi_{t t}^{S}+\alpha \varphi_{t}^{S}+\sin \varphi^{S} \cos \varphi^{I}=\frac{\left(\gamma_{A}+\gamma_{B}\right)(1-s)}{2(1+s)} .
\end{aligned}
$$

The $1 /(1 \pm s)$ coefficients of the second order space derivative give, respectively, the velocities $c^{+}$and $c^{-}$for the in-phase and out-of-phase solution ${ }^{9}$. Equation (3) shows that $\gamma_{A}+\gamma_{B}$ is the driving term for a symmetric excitation as was demonstrated experimentally in connection with Fig. 2. Equation (2) shows that $\gamma_{A}-\gamma_{B}$ is the generator of in-phase excitations. In our case $\varphi^{S}$ is a fluxon, giving a fluxonantifluxon state in the junctions, while $\varphi^{I}$ are oscillations generated by $\gamma_{A}-\gamma_{B}$; those oscillations are in phase in the two junctions. As the currents in the two junctions differ more and more, the amplitude of $\varphi^{I}$ increases and eventually causes the phase locking to disappear. The implication of Eqs. (2) and (3) is that the system should be considered as one system rather than two coupled.

Figure 4 shows a numerical solution of Eqs. (2) and (3); to make things clear we show the fluxon-fluxon excitation (arising for $\gamma_{B}<0$ ) i.e., $\varphi^{I}$ being a fluxon, and $\varphi^{S}$ being the waves. Parameters of the simulation are $l=7, \alpha=0.1$, $s=-0.66, \gamma_{A}=0.55$, and $\gamma_{B}=-0.35$. We show the time derivatives of the solutions. The top part shows the solutions for the phase derivatives independently; in the bottom part we summed and subtracted the phase derivatives to split the in-phase and out-of-phase components. Fluxons are the peaks in the derivatives. As can be seen, the solutions are composed of the sum of a fluxonic part and a small ampli- 


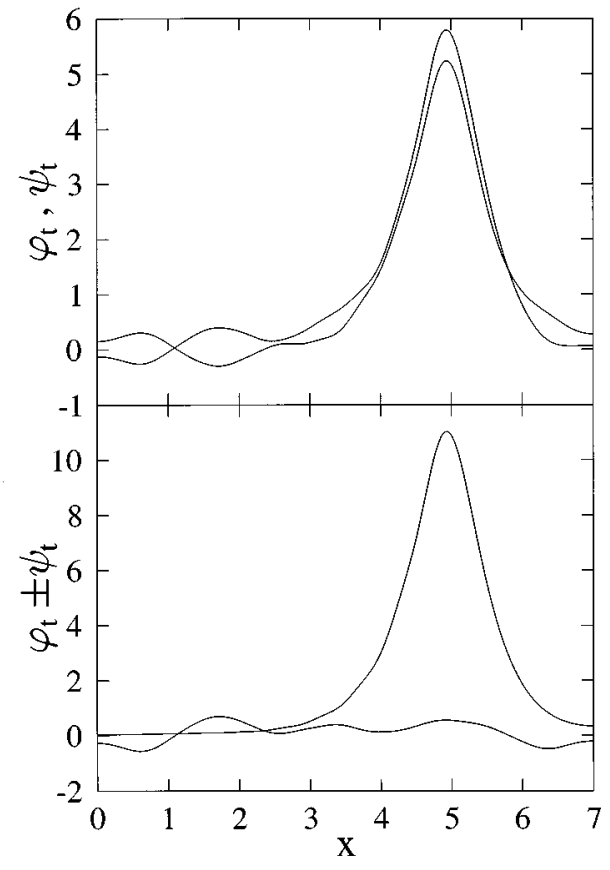

FIG. 4. Time derivatives of the solutions along the junctions. Top: one fluxon in each layer; bottom: bunched and symmetric components.

tude wave part. It is clearly seen in Fig. 4 that the wave is completely included in the out-of-phase part while the fluxons are included in the in-phase part.

In conclusion we have demonstrated the phase locking of a fluxon and an antifluxon in a stacked long Josephson junction. The zero field steps observed are consistent with theory and numerical simulations. We remark that, as a consequence of our model, the amplitude of the radiation emitted from the edges during the reflection of the fluxonantifluxon pair is expected to be other than zero, but when the junctions are biased with $\gamma_{A}=\gamma_{B}$, the fields emitted from the two junctions would cancel one another. In fact, we did observe radiation emission from our samples. Work is in progress on these and other aspects of fluxon dynamics observed in connection with these measurements.

Useful discussions with R. D. Parmentier are gratefully acknowledged. The help with the setup and data collection of M. H. Manscher and M. Nordahn has been invaluable. Two of the authors (G.C. and G.C.) thank the Department of Physics of the Technical University of Denmark for the warm hospitality.

\footnotetext{
${ }^{1}$ A. V. Ustinov, H. Kohlstedt, M. Cirillo, N. F. Pedersen, G. Hallmanns, and C. Heiden, Phys. Rev. B 48, 10614 (1993).

${ }^{2}$ H. Amin, M. G. Blamire, and J. E. Evetts, IEEE Trans. Appl. Supercond. 3, 2204 (1993).

${ }^{3}$ P. Barbara, A. V. Ustinov, and G. Costabile, Phys. Lett. A 191, 443 (1994).

${ }^{4}$ S. Sakai, A. V. Ustinov, H. Kohlstedt, A. Petraglia, and N. F. Pedersen, Phys. Rev. B 50, 12905 (1994).

${ }^{5}$ A. Petraglia, A. V. Ustinov, N. F. Pedersen, and S. Sakai, J. Appl. Phys. 77, 1171 (1995).

${ }^{6}$ S. Sakai, P. Bodin, and N. F. Pedersen, J. Appl. Phys. 73, 2411 (1993).

${ }^{7}$ R. Monaco, A. Polcari, and L. Capogna, J. Appl. Phys. 58, 3278 (1995).

${ }^{8}$ N. Gronbech-Jensen, D. Cai, and M. R. Samuelsen, Phys. Rev. B 48, 16160 (1993); N. Gronbech-Jensen, D. Cai, A. R. Bishop, A. W. Lau, and P. S. Lomdhal, ibid. 50, 6352 (1994).

${ }^{9}$ A. Petraglia, N. F. Pedersen, P. L. Christiansen, and A. V. Ustinov (unpublished)
} 